OPEN ACCESS

Edited by: James W. Grau,

Texas A\&M University, United States

Reviewed by:

Kyle Baumbauer,

University of Kansas Medical Center,

United States

Klaus Funke,

Ruhr University Bochum, Germany Mitchell Ryan Goldsworthy, University of Adelaide, Australia

*Correspondence: Monika Klírová monika.klirova@nudz.cz

Received: 14 December 2019 Accepted: 28 April 2020 Published: 29 May 2020

Citation: Klírová M, Hejzlar M, Kostýlková L,

Mohr P, Rokyta R and Novák $T$ (2020) Prolonged Continuous Theta Burst Stimulation of the Motor Cortex Modulates Cortical Excitability But not Pain Perception.

Front. Syst. Neurosci. 14:27. doi: 10.3389/fnsys.2020.00027

\section{Prolonged Continuous Theta Burst Stimulation of the Motor Cortex Modulates Cortical Excitability But not Pain Perception}

\author{
Monika Klírová 1,2*, Martin Hejzlar ${ }^{1,2}$, Lenka Kostýlková 1,2, Pavel Mohr ${ }^{1,2}$, Richard Rokyta ${ }^{3}$ \\ and Tomáš Novák ${ }^{1,2}$ \\ ${ }^{1}$ Clinical Centre, National Institute of Mental Health, Klecany, Czechia, ${ }^{2}$ Department of Psychiatry, Third Faculty of Medicine, \\ Charles University, Prague, Czechia, ${ }^{3}$ Department of Normal, Pathological and Clinical Physiology, Third Faculty of Medicine, \\ Charles University, Prague, Czechia
}

Over the past decade, theta-burst stimulation (TBS) has become a focus of interest in neurostimulatory research. Compared to conventional repetitive transcranial magnetic stimulation (rTMS), TBS produces more robust changes in cortical excitability (CE). There is also some evidence of an analgesic effect of the method. Previously published studies have suggested that different TBS parameters elicit opposite effects of TBS on CE. While intermittent TBS (iTBS) facilitates CE, continuous TBS (cTBS) attenuates it. However, prolonged TBS (pTBS) with twice the number of stimuli produces the opposite effect. In a double-blind, placebo-controlled, cross-over study with healthy subjects $(n=24)$, we investigated the effects of various pTBS (cTBS, iTBS, and placebo TBS) over the right motor cortex on CE and pain perception. Changes in resting motor thresholds (RMTs) and absolute motor-evoked potential (MEP) amplitudes were assessed before and at two time-points (0-5 min; 40-45 min) after pTBS. Tactile and thermal pain thresholds were measured before and 5 min after application. Compared to the placebo, prolonged cTBS (pcTBS) transiently increased MEP amplitudes, while no significant changes were found after prolonged iTBS. However, the facilitation of CE after pcTBS did not induce a parallel analgesic effect. We confirmed that pcTBS with twice the duration converts the conventional inhibitory effect into a facilitatory one. Despite the short-term boost of CE following pcTBS, a corresponding analgesic effect was not demonstrated. Therefore, the results indicate a more complex regulation of pain, which cannot be explained entirely by the modulation of excitability.

Keywords: theta-burst stimulation, TBS, rTMS, cortical excitability, motor evoked potentials, pain, perception

\section{INTRODUCTION}

Repetitive transcranial magnetic stimulation (rTMS) of the primary motor (M1) cortex modulates cortical excitability (CE) with subsequent neuroplastic changes observed in the stimulated area and its association areas, as well (Cárdenas-Morales et al., 2010). According to evidence-based guidelines, rTMS of the M1 cortex causes a significant clinical improvement in various neurological disorders, including neuropathic pain, fibromyalgia, post-acute stroke, and motor injury in Parkinson's disease (Lefaucheur et al., 2020). 
Recently, neurostimulatory research has become interested in theta-burst stimulation (TBS), a modification of high-frequency rTMS (HF-rTMS). There is evidence that TBS produces even more robust changes in $\mathrm{CE}$ than those observed in the conventional rTMS protocols (Gamboa et al., 2010; Suppa et al., 2016). Therefore, TBS offers the possibility to induce changes in $\mathrm{CE}$ with a more pronounced post-modulation effect in the regulation of corticospinal excitability and synaptic plasticity, with the potential to optimize clinical stimulation protocols (Gamboa et al., 2010).

TBS typically consists of bursts of three pulses at $30 \mathrm{~Hz}$ or $50 \mathrm{~Hz}$, repeated five times per second with 600 pulses in total. There are two different paradigms: intermittent TBS (iTBS) and continuous TBS (cTBS). While iTBS facilitates CE (Huang et al., 2005; Di Lazzaro et al., 2008; Suppa et al., 2008, 2016), cTBS attenuates it (Di Lazzaro et al., 2005; Huang et al., 2005; Suppa et al., 2008; Goldsworthy et al., 2013; Wischnewski and Schutter, 2015). However, a prolonged form of cTBS (pcTBS) with twice the number of stimuli (1,200 pulses) produces a facilitatory effect similar to that of iTBS (Gamboa et al., 2010). By contrast, facilitatory iTBS (iTBS) is converted into inhibitory when applied for a period twice as long, which is called prolonged iTBS (piTBS; Gamboa et al., 2010). Moreover, CE can also be differently modulated for the TBS frequency used $(30 \mathrm{~Hz}$ vs. $50 \mathrm{~Hz}$ ), whereby its effect depends on time (minutes after TBS application) and interindividual differences between subjects (Chung et al., 2016).

It has been suggested that the analgesic effects of HF-rTMS of M1 stimulation result from the changes in pain modulation systems related to the long-term changes of neuronal excitability initiated by stimulation-induced changes in CE (Moisset et al., 2016), whereas the analgesic effect of TBS on the M1 cortex is explained by changes in excitability and ongoing activity in the connected areas that subsequently affect CE in M1 (Suppa et al., 2016).

Stimulation-induced pain relief is mainly attributed to the modulation of pain processing at the level of inhibition of the emotional response regions, such as the dorsolateral prefrontal cortex and the anterior cingulate cortex (ACC). Also, several other mechanisms that function through various neural pathways have been implicated, including the pain descending inhibitory system, whose activation at the brain stem level may lead to the inhibition of nociceptive transmission in the dorsal horn (Garcia-Larrea and Peyron, 2007; Leung et al., 2009).

An analgesic effect of HF-rTMS has been demonstrated in both experimental (Summers et al., 2004; Yoo et al., 2006; Nahmias et al., 2009; Borckardt et al., 2011) and clinical (Lefaucheur et al., 2001; Hirayama et al., 2006; Passard et al., 2007) pain studies. Furthermore, it has also been validated by several reviews and meta-analyses (Leung et al., 2009; Galhardoni et al., 2015; Goudra et al., 2017; Guo et al., 2017; Lefaucheur et al., 2020).

Similarly, TBS as a novel HF-rTMS approach has shown an analgesic effect in several inhibitory cTBS studies with healthy subjects (Poreisz et al., 2008; Csifcsak et al., 2009; Torta et al., 2013; Dowdle et al., 2019), while facilitatory iTBS studies have yielded predominantly negative results (Antal and Paulus, 2010; Borckardt et al., 2011; Houzé et al., 2013). Interestingly, in clinical trials with chronic pain patients, both cTBS and iTBS failed to affect the pain threshold and induce an analgesic effect (Lefaucheur et al., 2012; Gaertner et al., 2018). Only subjective transient pain relief has been observed in studies that used facilitatory iTBS as a priming protocol before HF-rTMS (Lefaucheur et al., 2012) or administered iTBS alone (Kohútová et al., 2017; Kim et al., 2020).

Regarding pTBS, only two studies with healthy subjects using pcTBS examined the analgesic effect, with both yielding positive results (Moisset et al., 2015; De Martino et al., 2019) despite different cortical areas were targeted (M1 and prefrontal cortex, respectively).

Evidence of a correlation between a stimulation-induced change in cortical CE of the M1 cortex and a change in the thermal pain threshold is mostly based on HF-rTMS trials in patients with pain (Lefaucheur et al., 2006; Mhalla et al., 2011). The absence of a post-stimulatory change in CE in the presence of an analgesic effect confirmed in several studies in healthy subjects is usually explained by the principle of homeostatic metaplasticity (Turrigiano, 2008). Healthy subjects without impaired homeostatic regulation do not display any significant post-stimulatory changes in CE (Moisset et al., 2016). Nevertheless, post-stimulatory changes in CE in healthy subjects have been repeatedly demonstrated in past experimental, non-pain studies, especially with (p)TBS (Huang et al., 2005; Di Lazzaro et al., 2008; Suppa et al., 2008, 2016; Gamboa et al., 2010; Goldsworthy et al., 2013; Wischnewski and Schutter, 2015).

It is also assumed that the analgesic effect of stimulatory protocols, particularly inhibitory cTBS in healthy subjects, is a consequence of cortical downregulation, whereas, in patients with chronic pain, the facilitatory HF-rTMS /iTBS leading to upregulation of $\mathrm{CE}$ is effective in pain relief (Antal and Paulus, 2010). These opposite results may be explained by the difference in the processing of acute provoked pain in healthy subjects and chronic pain symptoms, which are associated with maladaptive neuroplastic processes (Antal and Paulus, 2010). Interestingly, pcTBS studies have also shown that facilitatory pcTBS results in analgesic effects in healthy subjects (Moisset et al., 2015; De Martino et al., 2019). Overall, the exact mechanisms of action of rTMS-induced analgesic effects remain unclear and depend on many stimulation parameters.

Previously documented effects of pTBS prompt further investigation. Several studies reported an analgesic effect of inhibitory cTBS, as well as analgesia induced by facilitatory pcTBS, although it is not expected to be effective in relieving pain in healthy subjects. Presumably, an inhibitory piTBS protocol would induce analgesia in healthy subjects similar to other inhibitory protocols, including cTBS. So far, no studies in healthy subjects have tested whether inhibitory piTBS can reduce pain sensitivity.

Our primary objective was to test the effect of various pTBS protocols on CE of the M1 area. A secondary objective of the study was to investigate whether these protocols demonstrate an effect on the perception of pain. The effects of two different 
protocols of active pTBS (pcTBS, piTBS) were compared to placebo stimulation (plcTBS).

\section{MATERIALS AND METHODS}

\section{Subjects}

Healthy volunteers (HV) of both sexes, aged between 18 and 45 years, free of pain during the past 6 months and without analgesic medication, were invited to participate. Exclusion criteria were current diagnosis or history of pain, organic brain disorder or injury, any other serious medical condition that may interfere with the rTMS/TBS administration (e.g., epilepsy, metallic plates in the head), pregnancy or breastfeeding, psychiatric disorder including history of a substanceinduced disorder, except for nicotine addiction, and sensory or motor impairment that would preclude participation in the study. All of the participants signed an informed consent form, following the latest version of the Declaration of Helsinki, the study protocol was approved by the Independent Ethics Committee of the National Institute of Mental Health, Klecany.

\section{Study Protocol (Experimental Design)}

The experiment was designed as a double-blind, placebocontrolled, cross-over study with three experimental phases, carried out in a random order, after a wash-out period of 3 weeks or longer (Figure 1A). Participants were randomly assigned to one of six different sequences of three TBS conditions (pcTBS, piTBS, or plcTBS), following William's design. To assure an equal number of subjects in each sequence, we used non-stratified blocked randomization with a block size of six, computergenerated (www.randomization.com). Each experimental session was carried out at the same time of day, on the same weekday. Participants were asked to abstain from caffeine for at least $4 \mathrm{~h}$ before the TBS session and refrain from alcohol and any medication for $24 \mathrm{~h}$ before the TBS administration.

Both the study participants and the evaluating physicians were blind to the stimulation conditions and parameters. Quantitative Sensory Tests (QST), including measurement of tactile and thermal pain thresholds with subsequent measurement of resting motor thresholds (RMTs) and motor-evoked potential (MEP) amplitudes at the level of individual RMT, were performed before each pTBS session. During the pTBS sessions, the subjective acceptability of an individual stimulatory protocol was assessed. MEP amplitudes at the level of individual RMT with subsequent measurement of RMT after each pTBS session were evaluated immediately at the end of the pTBS application and then $40 \mathrm{~min}$ after the session. Tactile threshold and thermal/pain threshold at the end of pTBS application were measured 5 min after each stimulation session. Following the completion of the experiment, the subjects stayed in an idle state for the next $2 \mathrm{~h}$. Subsequent stimulation sessions with a different stimulation protocol and an identical assessment schedule followed after 3, and 6 weeks, respectively (Figure 1B).

\section{pTBS Administration}

TBS was administered using a MagPro R30 stimulator (Magventure $^{\circledR}$, Inc., Denmark) with a cool-B65 A/P figureof-eight-shaped coil designed for double-blind research (the symmetrical design of the coil prevents the active vs. placebo side from being identified) with accessory two surface electrodes attached on the subject's head for placebo stimulation. Surface electrodes used to stimulate skin sensation for plcTBS were also attached to the head during the active stimulation, to obtain identical settings. The coil was oriented tangentially to the scalp and horizontally in the anterior-posterior direction, which proved to be more effective in terms of pain relief than lateromedial positioning (André-Obadia et al., 2008; Andre-Obadia et al., 2018). TBS was administered over the contralateral motor cortex (right M1 area), specifically to the site corresponding to the somatotopic location of the left hand (thenar). The cortical area was located with a cool-B65 figure-of-eight-shaped coil by targeting with a single TMS pulse that induced a contralateral MEP of maximum amplitude in the left thenar (abductor pollicis brevis) while obtaining EMG responses with the accessory MEP monitor (EMG MagPro R30 equipment, Magventure ${ }^{\circledR}$ ).

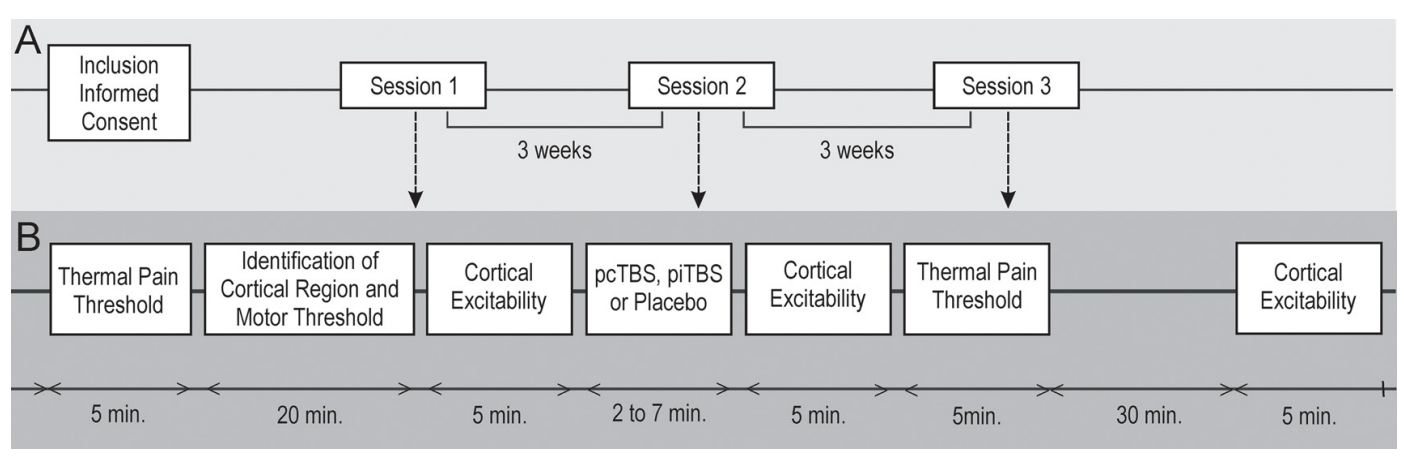

FIGURE 1 | Schematic overview of the whole study. A double-blind, cross-over study. Three prolonged theta-burst stimulation (pTBS) sessions (A) were carried out in random order with a minimum 3-week wash-out period. Healthy subjects $(n=24)$ were randomly assigned to a sequence of three pTBS conditions (B) over the right M1 cortex: continuous pTBS (pcTBS); intermittent pTBS (piTBS); placebo pTBS (plcTBS). Cortical excitability (CE) changes in resting motor thresholds (RMTs) and absolute motor-evoked potential (MEP) amplitudes were assessed before and at two time-intervals (0-5 min; 40-45 min) after pTBS. Tactile and thermal pain thresholds quantitative sensory tests (QST) were measured before and 5 min after pTBS application. 


\section{Parameter Settings}

pcTBS was applied with an intensity of 90\% RMT, three pulses at $30 \mathrm{~Hz}$ in repetition after $200 \mathrm{~ms}$ continuously, 1,200 pulses per session in total, 1 min and $20 \mathrm{~s}$ duration;

piTBS was applied with an intensity of $90 \%$ RMT, three pulses at $30 \mathrm{~Hz}$ in repetition after $200 \mathrm{~ms}$ intermittently within the interval of $1,800 \mathrm{~ms}$, with a train of 10 bursts and $8 \mathrm{~s}$ intertrain, 1,200 pulses per session in total, $6 \mathrm{~min}$ and $24 \mathrm{~s}$ duration;

plcTBS was applied with an A/P cool-B65 butterfly coil with an adjustable output for current stimulation of the subject's skin, producing a surface current synchronous with magnetic stimulation pulses. During the placebo protocol, the coil with oppositely oriented and simultaneously shielded magnetic stimulation pulses were applied to the same location as during the active session. Placebo current stimulation, generating similar sensory stimuli as the active stimulation protocols, was administered via two surface electrodes placed on the subject's right forehead and right temporal area.

\section{Assessments}

Motor CE was examined by single-pulse TMS paradigms, which included the measurement of RMT and MEP amplitudes at rest (Lefaucheur et al., 2012). The analgesic effect of TBS was measured with QST (Rolke et al., 2006), evaluating changes of pain threshold, specifically the changes in the thermal pain threshold. The measurement of the threshold for tactile sensation by von Frey testing (Johansson et al., 1980) enabled undisturbed mechanical sensitivity to be confirmed and is also a part of the QST.

All of the subjects enrolled in the study underwent measurement of CE (RMT, MEP amplitude) with a MagProR30 device and MEP Monitor accessory. The measurement was taken in a quiescent state avoiding active muscle contraction before using TBS. CE was assessed at the site corresponding to the somatotopic location (left thenar) of the stimulated area of the M1 cortex and was examined before and after each pTBS application and 40 min after each pTBS application, according to the protocol adapted from Moisset (Moisset et al., 2015). RMT was assessed as the lowest intensity, expressed as the percentage of maximum stimulatory output needed to elicit five or more electromyographic responses (EMG MagPro R30 equipment) $\geq 50 \mu \mathrm{V}$ within ten trials (Rossini et al., 1994). Individual values of MEP amplitudes within ten trials at the level of baseline individual RMT were measured and averaged (absolute MEP amplitude), and post-TBS MEP amplitudes were normalized by baseline average MEP amplitudes (normalized MEP amplitude).

All of the study subjects were also evaluated with QST that examined tactile and pain perception in the left (corresponding to the stimulated area of the M1 cortex) and right thenar eminences. They were assessed at the baseline and $5 \mathrm{~min}$ after each pTBS application (preceded by CE measurement). First, the tactile threshold was measured, then the thermal pain threshold was examined to eliminate the potential effect of the thermal generator on sensitivity disruption at the corresponding site. The tactile threshold was tested by the von Frey method (touchtest sensory evaluators, North Coast Medical). The thermal pain threshold was measured with a portable thermal stimulator of Algic stimuli (Yamamotová et al., 2017), a uniquely modified device that creates thermal stimulations with steadily increasing temperature $\left(20-70^{\circ} \mathrm{C}\right)$, until a participant indicates, by pressing the left button of a computer mouse, their first perception of the relevant sensation and then the first perception of pain (Summers et al., 2004).

After each stimulation, the study subjects also self-evaluated tolerability and acceptability (pain and general discomfort) of the different stimulation protocols, with a 10-point subjective Visual Analog Scale (VAS; from 0 representing the absence of pain or discomfort to 10 being unacceptable pain; Khedr et al., 2005).

\section{Statistical Analyses}

Subjective acceptability of TBS procedures assessed on a 10-point scale change across TBS conditions was compared by Friedman's test with consequent Wilcoxon's sign-ranked tests. The effect of TBS on CE parameters (absolute MEP amplitude and RMT) was calculated with repeated measures analysis of variance (RM ANOVA) including sequence as a between-subject factor, and treatment (piTBS, pcTBS, plcTBS) and time (baseline, immediately after, and after $40 \mathrm{mins}$ ) as within-subjects factors. For thermal thresholds, another within-subject factor, side, was added to the RM ANOVA model. Sphericity assumption was assessed by Mauchly's test and if a violation was detected, then degrees of freedom were adjusted using Huynh-Feldt correction. If there was a significant treatment $\times$ time interaction, simple main effects (one-way model) were calculated for time and treatment, and paired $t$-tests with Bonferroni's correction for multiple comparisons were consequently applied in the event of significant outcomes. For tactile threshold (ordinal scale) change comparisons, Friedmans ANOVA with Wilcoxons test was used. To assess inter-individual variability in MEP changes after TBS, the number of responders $(>10 \%$ increase from the baseline) and inverse responders (>10\% decrease from the baseline) was compared between conditions using Cochrane's Q test. Pearson's correlation coefficient was used to analyze relationships between the changes in thermal thresholds and normalized CE parameters (after/baseline). The thermal threshold changes between responders and non-responders, as well as between females and males under the respective TBS conditions, were compared using Welsh's $t$-test.

A sample size of 24 subjects should enable detecting effect size $\left(\eta_{\mathrm{P}}^{2}\right)$ of 0.14 or larger in the RM ANOVA model for withinsubjects simple main effects (time and treatment), at a given alpha of 0.05 , power (1-beta) of 0.90 , and estimated correlation of repeated measurements of 0.3 , if present.

All of the statistical analyses were performed using the STATISTICA 12 software (StatSoft, Inc., 2008). All of the tests were two-sided and $p<0.05$ was regarded as being statistically significant.

\section{RESULTS}

Twenty-four HV (mean age $30.4 \pm 3.3$ years, 11 females) were enrolled in the study and randomized to treatment sequences. Twenty-three subjects completed all three treatment conditions 
and were entered into further analyses. One subject dropped out of the study after pcTBS (female; sequence pcTBS $\rightarrow$ piTBS $\rightarrow$ plcTBS) due to procedural intolerance. The mean baseline RMT for the whole sample was $48.7 \pm 8.6 \%$ (range $35-66 \%$ ) of the maximum device output.

Both pTBS sessions were less tolerated than plcTBS; piTBS was less tolerated than pcTBS [VAS: piTBS: median 3 (IQR 1-6); pcTBS $1(0-3)$, plcTBS 0 (0-0); Friedman's ANOVA $\chi^{2}=22.1$, $p<0.001$; post hoc: piTBS vs. pcTBS; $z=3.52, p<0.001$ ]. Four subjects considered the pain felt during piTBS as being almost unbearable (VAS $\geq 7$ ). No other serious side effects were reported.

When analyzing the RMT change, RMANOVA revealed a significant treatment $\times$ time interaction $\left(F_{(4,68)}=2.76\right.$, $\left.p=0.03, \eta_{\mathrm{p}}^{2}=0.14\right)$, and main effect of time $\left(F_{(1.7,29.3)}=8.94\right.$, $\left.p=0.001, \eta_{\mathrm{p}}^{2}=0.34\right)$, but a non-significant effect of treatment $\left(F_{(1.9,31.7)}=1.14, p=0.33, \eta_{\mathrm{p}}^{2}=0.06\right)$. Neither effect of sequence $\left(F_{(5,17)}=1.62, p=0.22\right)$ nor sequence $\times$ treatment interaction $\left(F_{(9.1,31.7)}=1.13, p=0.37\right)$ was significant. Subsequent analysis indicated the only significant simple main effect of time for $\operatorname{pcTBS}(F=9.03, p=0.001)$ and Bonferroni's post hoc test showed a significant decrease in RMT for both post-TBS measurements (immediately: $-1.96,95 \% \mathrm{CI}-0.37$ to $-3.55, p=0.01 ; 40 \mathrm{~min}$ : $-2.61,-1.02$ to $-4.20, p<0.001)$ compared to the baseline. The simple main effects of treatment, however, only indicated a trend towards a significant difference between conditions after $40 \mathrm{~min}$ (immediately: $F=2.08, p=0.14 ; 40 \min : F=3.17, p=0.052$; Table 1, Figure 2A).

Analysis of absolute values of MEP amplitude at the level of the individual baseline RMT found significant treatment $\times$ time

TABLE 1 | Cortical excitability (absolute and normalized MEP amplitude, and $\mathrm{RMT}$ ), thermal thresholds and tactile thresholds after prolonged theta burst stimulation (pTBS).

\begin{tabular}{|c|c|c|c|c|}
\hline & & Baseline & After & 40 min after \\
\hline \multirow[t]{3}{*}{ Absolute MEP $(\mu \mathrm{V})$} & piTBS & $72.9 \pm 49.3$ & $75.7 \pm 57.7$ & $70.2 \pm 53.0$ \\
\hline & pcTBS & $69.0 \pm 47.1$ & $100.7 \pm 57.9^{\star \# \dagger}$ & $84.2 \pm 54.1$ \\
\hline & plcTBS & $73.0 \pm 53.4$ & $78.1 \pm 52.1$ & $75.8 \pm 53.4$ \\
\hline \multicolumn{5}{|l|}{ Normalized MEP1 } \\
\hline & piTBS & & $1.05 \pm 0.32$ & $0.98 \pm 0.33$ \\
\hline & pcTBS & & $1.61 \pm 0.71^{\# \dagger}$ & $1.29 \pm 0.53^{\dagger}$ \\
\hline & plcTBS & & $1.11 \pm 0.25$ & $1.07 \pm 0.34$ \\
\hline \multirow{3}{*}{$\begin{array}{l}\text { RMT (\% of device } \\
\text { output) }\end{array}$} & piTBS & $49.0 \pm 8.5$ & $48.4 \pm 8.7$ & $48.2 \pm 9.1$ \\
\hline & pcTBS & $49.2 \pm 8.8$ & $47.4 \pm 9.6^{\star}$ & $46.7 \pm 9.1^{\star}$ \\
\hline & plcTBS & $49.3 \pm 8.7$ & $49.0 \pm 8.6$ & $48.7 \pm 8.9$ \\
\hline
\end{tabular}

Thermal

threshold $\left({ }^{\circ} \mathrm{C}\right)^{2}$

$\begin{array}{llll} & \text { piTBS } & 47.1 \pm 3.3 & 47.0 \pm 3.9 \\ & \text { pcTBS } & 47.5 \pm 4.3 & 46.5 \pm 3.4 \\ & \text { plcTBS } & 46.0 \pm 3.6 & 46.7 \pm 2.8 \\ \text { Tactile threshold } & \text { piTBS } & 1.73 \pm 0.22 & 1.74 \pm 0.22 \\ (\mathbf{g})^{2} & & & \\ & \text { pcTBS } & 1.77 \pm 0.28 & 1.71 \pm 0.20 \\ & \text { plcTBS } & 1.73 \pm 0.22 & 1.71 \pm 0.17\end{array}$

Data are presented as mean $\pm S D$. piTBS: prolonged intermittent TBS; pcTBS: prolonged continuous TBS; plcTBS: placebo TBS. ${ }^{*} p<0.05$ vs. baseline; ${ }^{*} p<0.05$ vs. sham; ${ }^{\dagger} p<0.05$ vs. piTBS after Bonferroni's test. ${ }^{1}$ Normalized MEP-post-TBS MEP amplitudes normalized by baseline average MEP amplitudes. ${ }^{2}$ Data for thermal and tactile thresholds are presented as an average from both sides. interaction $\left(F_{(4,68)}=5.58, p<0.001, \eta_{\mathrm{p}}^{2}=0.25\right)$, and effect of time $\left(F_{(2,34)}=7.83, p=0.002, \quad \eta_{\mathrm{p}}^{2}=0.32\right)$, but no significant effect of treatment $\left(F_{(1.8,30.4)}=1.38, p=0.38\right.$, $\left.\eta_{\mathrm{p}}^{2}=0.05\right)$. The effects of sequence $\left(F_{(5,17)}=0.38, p=0.85\right)$, and sequence $\times$ treatment interaction $\left(F_{(8.9,30.4)}=0.71, p=0.69\right)$ were non-significant. Furthermore, the simple main effects of time revealed a significant increase in absolute MEP amplitude after pcTBS $(F=11.73, p<0.001)$, but not after either piTBS $(F=0.71 . p=0.50)$ or plcTBS $(F=0.66, p=0.52)$. Post hoc comparisons found a significantly increased absolute value of MEP amplitude after pcTBS (34.6, 95\%CI 16.8-52.3, $p<0.001$ ) but not after $40 \mathrm{~min}(17.3,-0.4$ to $35.1, p=0.058)$. The simple main effect of treatment was significant for measurement immediately after $(F=4.48, p=0.016)$, but not after $40 \mathrm{~min}$ $(F=1.03, p=0.36)$. The increase in MEP amplitudes after pcTBS was higher compared to both plcTBS (25.4, 1.8-49.0, $p=0.036)$ and piTBS $(28.0,4.4-51.7, p=0.02$; Table 1, Figure 2B).

Also, 6, 19, and 10 subjects were classified as responders ( $>10 \%$ increase of MEP after compared to before) to piTBS, pcTBS, and plcTBS, respectively (Cochran's $Q$ test: $Q=13.3$, $p=0.001$ ). A decrease in MEP amplitude $>10 \%$ (inverse responders) was found in 4,1 , and 5 and no change in 13,3 , and 8 subjects after piTBS, pcTBS, and the placebo, respectively.

In contrast to the CE parameters, no significant effect of TBS on thermal threshold was found (main effect of time $\left(F_{(1,17)}=0.77, p=0.39, \eta_{\mathrm{p}}^{2}=0.04\right)$, treatment $\left(F_{(2,34)}=0.64\right.$, $\left.p=0.53, \eta_{\mathrm{p}}^{2}=0.04\right)$, side $\left(F_{(1,17)}=2.62, p=0.12, \eta_{\mathrm{p}}^{2}=0.13\right)$, and treatment $\times$ time interaction $\left(F_{(2,34)}=0.71, p=0.50, \eta_{\mathrm{p}}^{2}=0.04\right.$, observed power of 0.13; Table 1, Figure 2C). Similarly, we failed to find any significant effect on tactile perception (Friedmans ANOVA: $\chi^{2}=1.47, d f=5, p=0.9$; Table 1).

Concerning the relationship between CE parameters and thermal thresholds, only a tendency toward a significant correlation between normalized MEP amplitude and thermal threshold change after piTBS was found $(r=-0.41$, $p=0.06)$. Otherwise, no significant correlation was revealed between changes in CE parameters and thermal threshold across the conditions (pcTBS $r=0.18, p=0.40$; plcTBS $r=0.07, p=0.77$ ). Furthermore, there was no difference in thermal threshold changes between responders and non-responders (piTBS: $t=1.26, p=0.23$; pcTBS: $t=0.83$, $p=0.46$; plcTBS: $t=0.81, p=0.43)$ or between genders (piTBS: $t=-0.66, p=0.52$; pcTBS: $t=-0.03, p=0.98$, $t=-0.10, p=0.92)$.

\section{DISCUSSION}

Our findings confirmed the effect of pcTBS on the facilitation of $\mathrm{CE}$, demonstrated by the increase of MEP amplitudes and the decrease of RMT. Compared to other stimulation protocols used in our study (piTBS and plcTBS), we found the pcTBS paradigm to be the only effective intervention. Moreover, the continuous form of pTBS was also the best tolerated active stimulation protocol. 

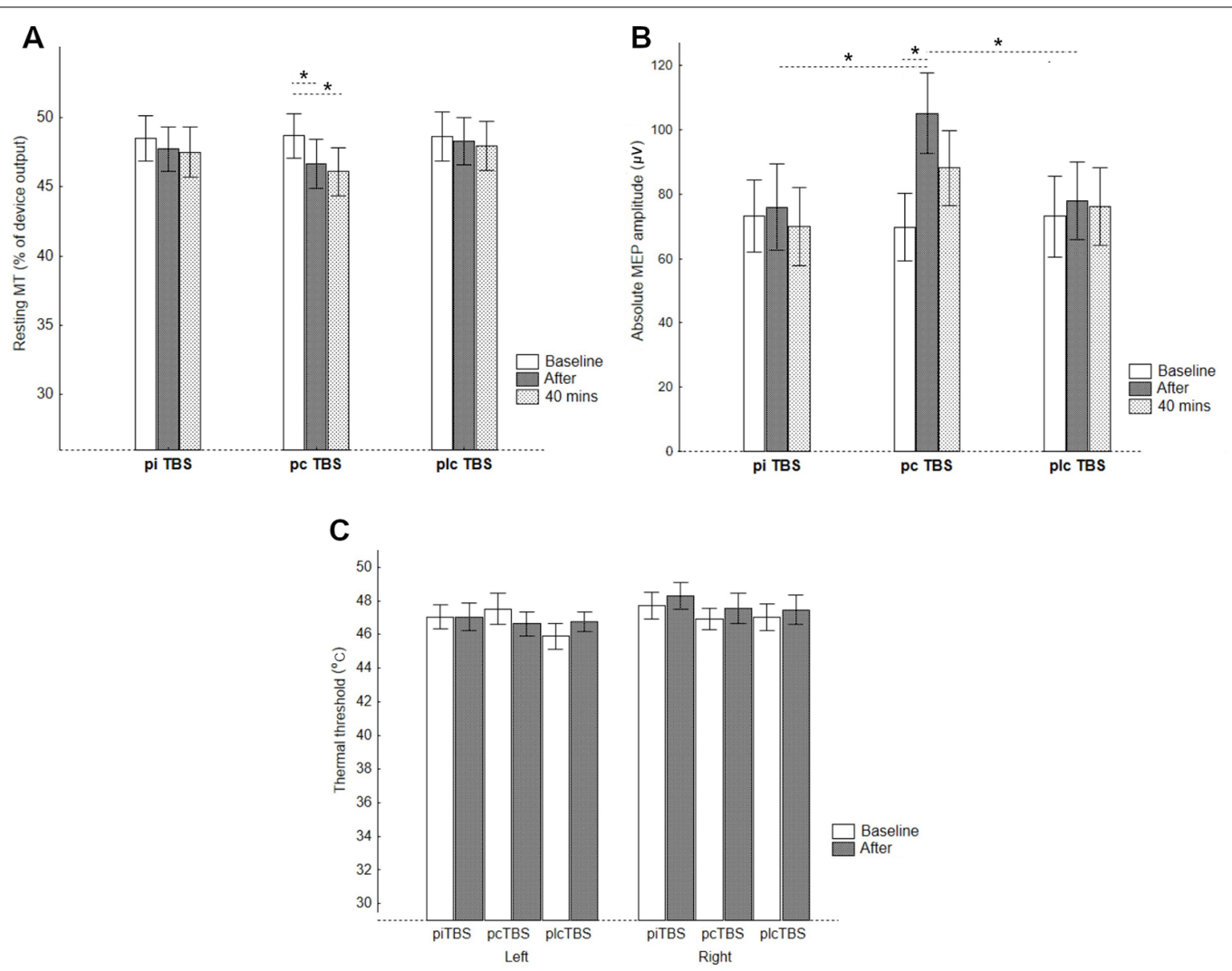

FIGURE 2 | CE represented by (A) RMTs, (B) absolute MEP amplitudes, and pain perception represented by (C) thermal thresholds for the thenar of the left and right thenar eminences after prolonged TBS (pTBS). pcTBS: continuous pTBS; piTBS: intermittent pTBS; plcTBS: placebo TBS. The data are presented as mean \pm SE. ${ }^{*} p<0.05$ after Bonferroni's test.

The results are fully congruent with the hypothesis that by doubling the stimulation duration of the prolonged form of cTBS, conventional inhibitory cTBS converts into facilitatory cTBS. The results are also in agreement with the results of a previously published study testing the effect of pTBS on CE (Gamboa et al., 2010). The use of a different frequency in our study $(30 \mathrm{~Hz}$ instead of $50 \mathrm{~Hz})$ did not change the outcome. On the other hand, piTBS did not affect any of the tested variables; therefore, we could not confirm the earlier observation that the piTBS would induce the change, i.e., immediate decrease CE in a group of healthy subjects (Gamboa et al., 2010). This could be attributed to the fact that, unlike Gamboa's study, we measured CE on RMT, but not on an active motor threshold (AMT).

Another possible explanation for CE facilitation after pcTBS, but not CE suppression after piTBS, maybe the fact that we were biased in detecting the potentially piTBS-induced CE suppression by measuring MEPs below the testable range. In an earlier study with non-prolonged TBS, the authors found that cTBS-induced MEP inhibition is observed by measuring at higher stimulus intensities (150\% RMT), while lower stimulus intensities (110\% RMT) are optimal for detecting iTBS-induced MEP facilitation (Goldsworthy et al., 2016). Based on their results, it may be assumed that potential piTBS-induced MEP inhibition will be observed when probed with a stimulus intensity much higher than at an RMT level.

However, our findings do not support the results reported in a previously published pTBS study with healthy subjects (Moisset et al., 2015). The authors failed to detect the change of CE after pcTBS but observed its analgesic effect. None of the pTBS protocols used in our study induced analgesia; specifically we did not observe any effect of pTBS on the thermal/pain threshold changes. Therefore, our results do not allow us to establish a relationship between the change of CE and analgesia induced by intracortical modulation with pTBS in healthy subjects. Nevertheless, different techniques used to measure pain threshold should be mentioned as a possible explanation for dissimilar findings compared to previous pain-related pTBS studies (Moisset et al., 2015; De Martino et al., 2019). Also, we cannot exclude type II error risk if true differences in thermal thresholds are moderate-sized.

There is a lack of rTMS/TBS studies in healthy subjects that examined the correlation between the change of $\mathrm{CE}$ and thermal/pain threshold changes. The correlation has been reported mostly in clinical rTMS trials of patients with pain (Lefaucheur et al., 2006; Mhalla et al., 2011). We assume that the effect of rTMS/TBS adjusts impaired thermal detection through the activation of systems that modulate the long-term change in 
neuronal excitability in patients with pain (Moisset et al., 2016). However, if the thermal sensation of healthy individuals is not disturbed, the correlation between the change of $\mathrm{CE}$ and the change of thermal threshold may not occur.

Recent contradictory data from TBS studies suggest that apart from the total number of pulses administered during the stimulation session, the varying effect (both on $\mathrm{CE}$ and pain threshold) may also be explained by the impact of other stimulation parameters (Goldsworthy et al., 2012). According to a TBS meta-analysis of non-prolonged cTBS (pcTBS) studies (using 600 pulses per session) that compared the effect of different stimulation frequencies $(30 \mathrm{~Hz}$ vs. $50 \mathrm{~Hz}$ ) on MEP suppression, the changes in the $30 \mathrm{~Hz}$ TBS subgroup were more persistent (Chung et al., 2016).

Besides, we cannot ignore the fact that other interindividual differences, such as the influence of BDNF polymorphism on the change of the amplitude of MEP after rTMS/TBS (Jacobs et al., 2014; Chung et al., 2016) may also play an important role in the effect of TBS (Jannati et al., 2017). Therefore, investigation of repeated measures within an individual may produce more reliable results than interindividual group comparisons (Suppa et al., 2016).

There are several possible sources of heterogeneity among experimentally-induced pain rTMS studies to be considered. It is supposed that various rTMS paradigms affect A-deltafiber and C-fiber-mediated induced pain in different ways (Leo and Latif, 2007). Also, stimulation-induced changes in pain perception in some rTMS (Summers et al., 2004; Lefaucheur et al., 2010; de Andrade et al., 2011) and TBS (Csifcsak et al., 2009; Torta et al., 2013; Moisset et al., 2015; De Martino et al., 2019) studies may be explained by the various measurement methods used. Laser and cold stimulation are standard methods in measuring pain perception. While acute laser-induced pain is mediated by activation of the A-deltafiber pathway, cold-induced pain is mediated by a combination of the A-delta-fiber and C-fiber pathways (Leo and Latif, 2007). Moreover, the thermal stimulator, with the gradually induced heat pain used in our study, presumably employs a mechanism of activation mediated by both the A-delta-fiber and the C-fiber pathways (Kostek et al., 2016). Therefore, it can be assumed that our measurement of the pain threshold adequately reflects previous (p)TBS studies (Moisset et al., 2015; De Martino et al., 2019).

The main limitation of our study is that, unlike other pTBS studies (Moisset et al., 2015; De Martino et al., 2019), we did not include non-pcTBS or conventional HF-rTMS as a positive control to verify the analgesic effect previously demonstrated by HF-rTMS/cTBS studies (Summers et al., 2004; Yoo et al., 2006; Nahmias et al., 2009; Borckardt et al., 2011). However, for testing stimulation-induced changes of CE, HF-rTMS would not be appropriate as a positive control since previous studies showed that the after-effects of rTMS-induced change in CE were highly variable and inhomogeneous among individuals (Maeda et al., 2000; Pell et al., 2011).

Similarly to previous pTBS studies (Moisset et al., 2015; De Martino et al., 2019), we determined CE based on RMT, while AMT would be a better criterion for adjusting the strength of TBS application (Huang et al., 2005; Gamboa et al., 2010; Lefaucheur et al., 2012). This is another limitation of our study, since AMT more reliably sets a defined state, while RMT may vary greatly according to the subject's attention in motor preparation and spatial attention (Mars et al., 2007).

We should point out that the determination of $\mathrm{CE}$ based on RMT, levels of MEP amplitude, is a rather indirect measure. Currently, TMS-evoked EEG responses are preferred as a more direct measure of CE since the RMT and MEP amplitudes may differ for other reasons than changes in CE. Moreover, the aftereffects of rTMS and TBS are highly variable between individuals (Hamada et al., 2013).

Another limitation of our study is the absence of a neuronavigation system to target the area for stimulation according to the structural/functional imaging examination (specifically MRI, fMRI). In general, neuronavigated rTMS/TBS allows a better reproducibility and accuracy regarding the definition of the stimulation and potentially greater efficacy of the method (Moisset et al., 2016). It should be noted that the accuracy of the functional targeting with the coil focused on the specific M1 area (corresponding to the somatotopic location of the left hand), that enables electromyographic responses of the corresponding muscles to be immediately detected, is similar to focusing with neuronavigation (Herwig et al., 2002).

Current knowledge suggests that pain relief induced by HF-rTMS and TBS is mainly attributed to induced changes in neuronal excitability in the areas associated with emotional pain processing, particularly the ACC modulation. Therefore, it would be of interest to test the effect of (p)TBS by deep stimulation (e.g., with H-coil) targeted directly to the rostral part of the ACC and to investigate its potential analgesic effect.

In conclusion, following previously published research documenting pcTBS-induced changes in CE of the M1 cortex, our results suggest that pcTBS represents a well-tolerated, non-invasive method that increases $\mathrm{CE}$. We confirmed that a prolonged form of cTBS with twice the duration converts the conventional inhibitory effect into a facilitatory one. In contrast to previous studies, we failed to demonstrate the analgesic effect of pcTBS. Further studies are needed to clarify these contradictory findings. Our results indicate more complex regulation of pain that cannot be explained entirely by the modulation of M1 excitability. Future studies, especially with TMS-evoked EEG responses, should examine pTBS-induced changes in neuronal excitability in associated brain areas that are responsible for pain processing.

\section{DATA AVAILABILITY STATEMENT}

The datasets generated for this study are available on request to the corresponding author.

\section{ETHICS STATEMENT}

The studies involving human participants were reviewed and approved by Independent Ethics Committee (IEC) of the National Institute of Mental Health, Klecany, Czechia. The 
patients/participants provided their written informed consent to participate in this study.

\section{AUTHOR CONTRIBUTIONS}

MK contributed to the study conception and design analysis, data collection, interpretation of results, and drafting and revision of the article. $\mathrm{MH}$ contributed to data acquisition and assembly and interpretation of the data. LK contributed to the acquisition, assembly, and analysis of the data. PM contributed to the conception and design, and drafting

\section{REFERENCES}

Andre-Obadia, N., Magnin, M., Simon, E., and Garcia-Larrea, L. (2018). Somatotopic effects of rTMS in neuropathic pain? A comparison between stimulation over hand face motor areas. Eur. J. Pain 22, 707-715. doi: 10.1002/ejp.1156

André-Obadia, N., Mertens, P., Gueguen, A., Peyron, R., and Garcia-Larrea, L. (2008). Pain relief by rTMS. Differential effect of current flow but no specific action on pain subtypes. Neurology 71, 833-840. doi: 10.1212/01.wnl. 0000325481.61471.f0

Antal, A., and Paulus, W. (2010). Effects of transcranial theta-burst stimulation on acute pain perception. Restor. Neurol. Neurosci. 28, 477-484. doi: 10.3233/rnn2010-0555

Borckardt, J. J., Reeves, S. T., Beam, W., Jensen, M. P., Gracely, R. H., Katz, S., et al. (2011). A randomized, controlled investigation of motor cortex transcranial magnetic stimulation (TMS) effects on quantitative sensory measures in healthy adults: evaluation of TMS device parameters. Clin. J. Pain 27, 486-494. doi: 10.1097/ajp.0b013e31820d2733

Cárdenas-Morales, L., Nowak, D. A., Kammer, T., Wolf, R. C., and SchönfeldtLecuona, C. (2010). Mechanisms and applications of theta-burst rTMS on the human motor cortex. Brain Topogr. 22, 294-306. doi: 10.1007/s10548-0090084-7

Chung, S. W., Hill, A. T., Rogasch, N. C., Hoy, K. E., and Fitzgerald, P. B. (2016). Use of theta-burst stimulation in changing excitability of motor cortex: a systematic review and meta-analysis. Neurosci. Biobehav. Rev. 63, 43-64. doi: 10.1016/j.neubiorev.2016.01.008

Csifcsak, G., Nitsche, M. A., Baumgärtner, U., Paulus, W., Treede, R.-D., and Antal, A. (2009). Electrophysiological correlates of reduced pain perception after theta-burst stimulation. Neuroreport 20, 1051-1055. doi: 10.1097/wnr. 0b013e32832e0c74

de Andrade, D. C., Mhalla, A., Adam, F., Texeira, M. J., and Bouhassira, D. (2011). Neuropharmacological basis of rTMS-induced analgesia: the role of endogenous opioids. Pain 152, 320-326. doi: 10.1016/j.pain.2010.10.032

De Martino, E., Fernandes, A. M., Galhardoni, R., Souza, C. D. O., De Andrade, D. C., and Graven-Nielsen, T. (2019). Sessions of prolonged continuous theta burst stimulation or high-frequency $10 \mathrm{~Hz}$ stimulation to left dorsolateral prefrontal cortex for 3 days decreased pain sensitivity by modulation of the efficacy of conditioned pain modulation. J. Pain 20, 1459-1469. doi: 10.1016/j.jpain.2019.05.010

Di Lazzaro, V., Pilato, F., Dileone, M., Profice, P., Oliviero, A., Mazzone, P., et al. (2008). The physiological basis of the effects of intermittent theta burst stimulation of the human motor cortex. J. Physiol. 586, 3871-3879. doi: 10.1113/jphysiol.2008.152736

Di Lazzaro, V., Pilato, F., Saturno, E., Oliviero, A., Dileone, M., Mazzone, P., et al. (2005). Theta-burst repetitive transcranial magnetic stimulation suppresses specific excitatory circuits in the human motor cortex. J. Physiol. 565, 945-950. doi: $10.1113 /$ jphysiol.2005.087288

Dowdle, L., Imperatore, J., Hamilton, S., George, M., Borckardt, J., and Hanlon, C. (2019). Attenuating pain with theta burst stimulation (TBS): a sham-controlled neuroimaging study evaluating the relative efficacy of medial versus dorsolateral stimulation. Brain Stimul. 12, 531-532. doi: 10.1016/j.brs. 2018.12.751 and revision of the article. RR contributed to conception and design, interpretation of data, and revision of the article. TN contributed to conception and design analysis of data, interpretation of data, and drafting and revision of the article.

\section{FUNDING}

The study was supported by Grant No. 16-31380A of the Ministry of Health, Czechia, and project No. LO1611 with a financial support from the MEYS under the NPU I program, Czechia.

Gaertner, M., Kong, J. T., Scherrer, K. H., Foote, A., Mackey, S., and Johnson, K. A. (2018). Advancing transcranial magnetic stimulation methods for complex regional pain syndrome: an open-label study of paired theta burst and high-frequency stimulation. Neuromodulation 21, 409-416. doi: 10.1111/ner. 12760

Galhardoni, R., Correia, G. S., Araujo, H., Yeng, L. T., Fernandes, D. T., Kaziyama, H. H., et al. (2015). Repetitive transcranial magnetic stimulation in chronic pain: a review of the literature. Arch. Phys. Med. Rehabil. 96, S156-S172. doi: 10.1016/j.apmr.2014.11.010

Gamboa, O. L., Antal, A., Moliadze, V., and Paulus, W. (2010). Simply longer is not better: reversal of theta burst after-effect with prolonged stimulation. Exp. Brain Res. 204, 181-187. doi: 10.1007/s00221-010-2293-4

Garcia-Larrea, L., and Peyron, R. (2007). Motor cortex stimulation for neuropathic pain: from phenomenology to mechanisms. NeuroImage 37, S71-S79. doi: 10.1016/j.neuroimage.2007.05.062

Goldsworthy, M. R., Pitcher, J. B., and Ridding, M. C. (2012). A comparison of two different continuous theta burst stimulation paradigms applied to the human primary motor cortex. Clin. Neurophysiol. 123, 2256-2263. doi: 10.1016/j. clinph.2012.05.001

Goldsworthy, M. R., Pitcher, J. B., and Ridding, M. C. (2013). Neuroplastic modulation of inhibitory motor cortical networks by spaced theta burst stimulation protocols. Brain Stimul. 6, 340-345. doi: 10.1016/j.brs.2012.06.005

Goldsworthy, M. R., Vallence, A.-M., Hodyl, N. A., Semmler, J. G., Pitcher, J. B., and Ridding, M. C. (2016). Probing changes in corticospinal excitability following theta burst stimulation of the human primary motor cortex. Clin. Neurophysiol. 127, 740-747. doi: 10.1016/j.clinph.2015.06.014

Goudra, B., Shah, D., Balu, G., Gouda, G., Balu, A., Borle, A., et al. (2017) Repetitive transcranial magnetic stimulation in chronic pain: a meta-analysis. Anesth. Essays Res. 11, 751-757. doi: 10.4103/aer.AER_10_17

Guo, Q., Li, C., and Wang, J. (2017). Updated review on the clinical use of repetitive transcranial magnetic stimulation in psychiatric disorders. Neurosci. Bull. 33 , 747-756. doi: 10.1007/s12264-017-0185-3

Hamada, M., Murase, N., Hasan, A., Balaratnam, M., and Rothwell, J. C. (2013). The role of interneuron networks in driving human motor cortical plasticity. Cereb. Cortex 23, 1593-1605. doi: 10.1093/cercor/bhs147

Herwig, U., Kölbel, K., Wunderlich, A. P., Thielscher, A., Von Tiesenhausen, C., Spitzer, M., et al. (2002). Spatial congruence of neuronavigated transcranial magnetic stimulation and functional neuroimaging. Clin. Neurophysiol. 113, 462-468. doi: 10.1016/s1388-2457(02)00026-3

Hirayama, A., Saitoh, Y., Kishima, H., Shimokawa, T., Oshino, S., Hirata, M., et al. (2006). Reduction of intractable deafferentation pain by navigation-guided repetitive transcranial magnetic stimulation of the primary motor cortex. Pain 122, 22-27. doi: 10.1016/j.pain.2005.12.001

Houzé, B., Bradley, C., Magnin, M., and Garcia-Larrea, L. (2013). Changes in sensory hand representation and pain thresholds induced by motor cortex stimulation in humans. Cereb. Cortex 23, 2667-2676. doi: 10.1093/cercor/bhs255

Huang, Y.-Z., Edwards, M. J., Rounis, E., Bhatia, K. P., and Rothwell, J. C. (2005). Theta burst stimulation of the human motor cortex. Neuron 45, 201-206. doi: 10.1016/j.neuron.2004.12.033

Jacobs, M. F., Tsang, P., Lee, K. G., Asmussen, M. J., Zapallow, C. M., and Nelson, A. J. (2014). $30 \mathrm{~Hz}$ theta-burst stimulation over primary somatosensory 
cortex modulates corticospinal output to the hand. Brain Stimul. 7, 269-274. doi: 10.1016/j.brs.2013.12.009

Jannati, A., Block, G., Oberman, L. M., Rotenberg, A., and PascualLeone, A. (2017). Interindividual variability in response to continuous thetaburst stimulation in healthy adults. Clin. Neurophysiol. 128, 2268-2278. doi: 10.1016/j.clinph.2017.08.023

Johansson, R., Vallbo, A., and Westling, G. (1980). Thresholds of mechanosensitive afferents in the human hand as measured with von Frey hairs. Brain Res. 184, 343-351. doi: 10.1016/0006-8993(80)90803-3

Khedr, E. M., Kotb, H., Kamel, N., Ahmed, M., Sadek, R., and Rothwell, J. (2005). Longlasting antalgic effects of daily sessions of repetitive transcranial magnetic stimulation in central and peripheral neuropathic pain. J. Neurol. Neurosurg. Psychiatry 76, 833-838. doi: 10.1136/jnnp.2004.055806

Kim, J. K., Park, H. S., Bae, J. S., Jeong, Y. S., Jung, K. J., and Lim, J. Y. (2020). Effects of multi-session intermittent theta burst stimulation on central neuropathic pain: a randomized controlled trial. NeuroRehabilitation 46, 127-134. doi: 10.3233/nre-192958

Kohútová, B., Fricová, J., Klírová, M., Novák, T., and Rokyta, R. (2017). Theta burst stimulation in the treatment of chronic orofacial pain: a randomized controlled trial. Physiol. Res. 66, 1041-1047. doi: 10.33549/physiolres.933474

Kostek, M., Polaski, A., Kolber, B., Ramsey, A., Kranjec, A., and Szucs, K. (2016). A protocol of manual tests to measure sensation and pain in humans. J. Vis. Exp. 118:e54130. doi: 10.3791/54130

Lefaucheur, J.-P., Aleman, A., Baeken, C., Benninger, D. H., Brunelin, J., Di Lazzaro, V., et al. (2020). Evidence-based guidelines on the therapeutic use of repetitive transcranial magnetic stimulation (rTMS): an update(2014-2018). Clin. Neurophysiol. 131, 474-528. doi: 10.1016/j.clinph.2019.11.002

Lefaucheur, J. P., Ayache, S., Sorel, M., Farhat, W., Zouari, H., De Andrade, D. C., et al. (2012). Analgesic effects of repetitive transcranial magnetic stimulation of the motor cortex in neuropathic pain: influence of theta burst stimulation priming. Eur. J. Pain 16, 1403-1413. doi: 10.1002/j.1532-2149.2012.00150.x

Lefaucheur, J.-P., Drouot, X., Keravel, Y., and Nguyen, J.-P. (2001). Pain relief induced by repetitive transcranial magnetic stimulation of precentral cortex. Neuroreport 12, 2963-2965. doi: 10.1097/00001756-200109170-00041

Lefaucheur, J., Drouot, X., Menard-Lefaucheur, I., Keravel, Y., and Nguyen, J. (2006). Motor cortex rTMS restores defective intracortical inhibition in chronic neuropathic pain. Neurology 67, 1568-1574. doi: 10.1212/01.wnl.0000242731. $10074.3 \mathrm{c}$

Lefaucheur, J.-P., Jarry, G., Drouot, X., Ménard-Lefaucheur, I., Keravel, Y., and Nguyen, J.-P. (2010). Motor cortex rTMS reduces acute pain provoked by laser stimulation in patients with chronic neuropathic pain. Clin. Neurophysiol. 121, 895-901. doi: 10.1016/j.clinph.2009.12.028

Leo, R. J., and Latif, T. (2007). Repetitive transcranial magnetic stimulation (rTMS) in experimentally induced and chronic neuropathic pain: a review. J. Pain 8, 453-459. doi: 10.1016/j.jpain.2007.01.009

Leung, A., Donohue, M., Xu, R., Lee, R., Lefaucheur, J.-P., Khedr, E. M., et al. (2009). rTMS for suppressing neuropathic pain: a meta-analysis. J. Pain 10, 1205-1216. doi: 10.1016/j.jpain.2009.03.010

Maeda, F., Keenan, J. P., Tormos, J. M., Topka, H., and Pascual-Leone, A. (2000). Interindividual variability of the modulatory effects of repetitive transcranial magnetic stimulation on cortical excitability. Exp. Brain Res. 133, 425-430. doi: $10.1007 /$ s002210000432

Mars, R. B., Bestmann, S., Rothwell, J. C., and Haggard, P. (2007). Effects of motor preparation and spatial attention on corticospinal excitability in a delayedresponse paradigm. Exp. Brain Res. 182, 125-129. doi: 10.1007/s00221-0071055-4

Mhalla, A., Baudic, S., De Andrade, D. C., Gautron, M., Perrot, S., Teixeira, M. J., et al. (2011). Long-term maintenance of the analgesic effects of transcranial magnetic stimulation in fibromyalgia. Pain 152, 1478-1485. doi: 10.1016/j.pain. 2011.01.034

Moisset, X., De Andrade, D. C., and Bouhassira, D. (2016). From pulses to pain relief: an update on the mechanisms of rTMS-induced analgesic effects. Eur. J. Pain 20, 689-700. doi: 10.1002/ejp.811

Moisset, X., Goudeau, S., Poindessous-Jazat, F., Baudic, S., Clavelou, P., and Bouhassira, D. (2015). Prolonged continuous theta-burst stimulation is more analgesic than 'classical' high frequency repetitive transcranial magnetic stimulation. Brain Stimul. 8, 135-141. doi: 10.1016/j.brs.2014.10.006
Nahmias, F., Debes, C., De Andrade, D. C., Mhalla, A., and Bouhassira, D. (2009). Diffuse analgesic effects of unilateral repetitive transcranial magnetic stimulation (rTMS) in healthy volunteers. Pain 147, 224-232. doi: 10.1016/j. pain.2009.09.016

Passard, A., Attal, N., Benadhira, R., Brasseur, L., Saba, G., Sichere, P., et al. (2007). Effects of unilateral repetitive transcranial magnetic stimulation of the motor cortex on chronic widespread pain in fibromyalgia. Brain 130, 2661-2670. doi: 10.1093/brain/awm189

Pell, G. S., Roth, Y., and Zangen, A. (2011). Modulation of cortical excitability induced by repetitive transcranial magnetic stimulation: influence of timing and geometrical parameters and underlying mechanisms. Prog. Neurobiol. 93, 59-98. doi: 10.1016/j.pneurobio.2010.10.003

Poreisz, C., Csifcsák, G., Antal, A., Levold, M., Hillers, F., and Paulus, W. (2008). Theta burst stimulation of the motor cortex reduces laser-evoked pain perception. Neuroreport 19, 193-196. doi: 10.1097/wnr.0b013e328 $2 \mathrm{f} 45498$

Rolke, R., Magerl, W., Campbell, K. A., Schalber, C., Caspari, S., Birklein, F., et al. (2006). Quantitative sensory testing: a comprehensive protocol for clinical trials. Eur. J. Pain 10, 77-77. doi: 10.1016/j.ejpain.2005.02.003

Rossini, P. M., Barker, A., Berardelli, A., Caramia, M., Caruso, G., Cracco, R., et al. (1994). Non-invasive electrical and magnetic stimulation of the brain, spinal cord and roots: basic principles and procedures for routine clinical application. Report of an IFCN committee. Electroencephalogr. Clin. Neurophysiol. 91, 79-92. doi: 10.1016/0013-4694(94)90029-9

Summers, J., Johnson, S., Pridmore, S., and Oberoi, G. (2004). Changes to cold detection and pain thresholds following low and high frequency transcranial magnetic stimulation of the motor cortex. Neurosci. Lett. 368, 197-200. doi: 10.1016/j.neulet.2004.07.008

Suppa, A., Huang, Y.-Z., Funke, K., Ridding, M., Cheeran, B., Di Lazzaro, V., et al. (2016). Ten years of theta burst stimulation in humans: established knowledge, unknowns and prospects. Brain Stimul. 9, 323-335. doi: 10.1016/j.brs.2016. 01.006

Suppa, A., Ortu, E., Zafar, N., Deriu, F., Paulus, W., Berardelli, A., et al. (2008). Theta burst stimulation induces after-effects on contralateral primary motor cortex excitability in humans. J. Physiol. 586, 4489-4500. doi: 10.1113/jphysiol. 2008.156596

StatSoft, Inc. (2008). STATISTICA (data analysis software system), version 12 . Available online at: www.statsoft.com.

Torta, D. M., Legrain, V., Algoet, M., Olivier, E., Duque, J., and Mouraux, A. (2013). Theta burst stimulation applied over primary motor and somatosensory cortices produces analgesia unrelated to the changes in nociceptive event-related potentials. PLoS One 8:e73263. doi: 10.1371/journal.pone. 0073263

Turrigiano, G. G. (2008). The self-tuning neuron: synaptic scaling of excitatory synapses. Cell 135, 422-435. doi: 10.1016/j.cell.2008.10.008

Wischnewski, M., and Schutter, D. J. (2015). Efficacy and time course of theta burst stimulation in healthy humans. Brain Stimul. 8, 685-692. doi: 10.1016/j.brs. 2015.03.004

Yamamotová, A., Hrabak, P., Hříbek, P., and Rokyta, R. (2017). Do multiple body modifications alter pain threshold? Physiol. Res. 66, S493-S500. doi: $10.33549 /$ physiolres.933804

Yoo, W.-K., Kim, Y.-H., Doh, W.-S., Lee, J.-H., Jung, K.-I., Park, D.-S., et al. (2006). Dissociable modulating effect of repetitive transcranial magnetic stimulation on sensory and pain perception. Neuroreport 17, 141-144. doi: 10.1097/01.wnr. 0000198438.37012.d6

Conflict of Interest: The authors declare that the research was conducted in the absence of any commercial or financial relationships that could be construed as a potential conflict of interest.

Copyright (C) 2020 Klírová, Hejzlar, Kostýlková, Mohr, Rokyta and Novák. This is an open-access article distributed under the terms of the Creative Commons Attribution License (CC BY). The use, distribution or reproduction in other forums is permitted, provided the original author(s) and the copyright owner(s) are credited and that the original publication in this journal is cited, in accordance with accepted academic practice. No use, distribution or reproduction is permitted which does not comply with these terms. 